\title{
UNDOMINATED (AND) PERFECT EQUILIBRIA IN POISSON GAMES*
}

\author{
FRANCESCO DE SINOPOLI ${ }^{\dagger}$ AND CARLOS GONZÁLEZ PIMIENTA ${ }^{\dagger, \ddagger}$
}

\begin{abstract}
In games with population uncertainty some perfect equilibria are in dominated strategies. We prove that every Poisson game has at least one perfect equilibrium in undominated strategies.
\end{abstract}

\section{INTRODUCTION}

Models of population uncertainty have been introduced by Myerson (1998, 2000) and Milchtaich (2004), in order to describe situations in which players do not know the number of opponents. Among these games, a special attention has been reserved to Poisson games, where the number of players is a Poisson random variable with a given mean and where the players' types are independent identically distributed random variables. The properties of the Poisson distribution make Poisson games an extremely convenient subclass of games. They are characterized by the properties of independent actions (for every possible strategy profile the number of players who take different actions are independent random variables) and environmental equivalence (a player assesses the same probability for the type profile of the others as

$\dagger$ Department of Economics, Universidad Carlos III De Madrid, Calle Madrid, 126, 28903 Getafe (Madrid), SPAIN., TEl + 34916249594 , FAX +34916249329.

$\ddagger$ School of Economics, University of New South Wales, Sydney 2052, Australia.

E-mail addresses: fsinopol@eco.uc3m.es, jcgonzal@eco.uc3m.es.

Date: September 17, 2007.

JEL Classification. C72.

Key words and phrases. Poisson games, undominated equilibria, perfect equilibria.

Correspondence to: Francesco De Sinopoli.

* Preliminary discussions with Jean-François Mertens are gratefully acknowledged. We thank Luis Corchón and Giovanna Iannantuoni for helpful comments. Francesco thanks the Ramon y Cajal Fellowship, and acknowledges research funding from the Spanish MEC, Grant SEJ2006-11665-C02-0. Carlos thanks the Spanish MEC FPI Grant BES-2003-0822. The usual disclaimer applies. 
an external observer does for the type profile of the whole game, where a type profile is a vector that lists how many players there are of each type).

Myerson (1998) extends the definition of Nash equilibrium and shows its existence. The existing literature on equilibrium refinements in noncooperative game theory warns that we should be cautious about the strategic stability of the Nash equilibrium concept. If this concern is well founded, we can ask which Nash equilibria are self-enforcing in this setting.

The following example serves us to both introducing Poisson games to the reader and illustrating the nature of the question. A player is sitting at home and faces two possible alternatives, either she goes out to some social event, or she stays home. She does not know how many players are facing this same disjunctive, but she knows that this number is a Poisson random variable with parameter $n$. If she goes out and meets somebody she receives a payoff equal to 1 . If she meets nobody or decides to stay home, she gets a payoff equal to 0. Every player faces this same two options and has the same preferences.

The strategy "everybody stays home" is a Nash equilibrium of the described game. However, we cannot consider it a good equilibrium since players use a dominated strategy. It is not difficult to come up with similar examples with patently implausible Nash equilibria. ${ }^{1}$

Recall that in conventional normal form games (from now on just normal form games), a modest refinement like perfection only selects undominated strategies. This is the case in the previous example. However, in Poisson games this is not true in general. We can go further, straightforward extensions of proper and strictly perfect equilibrium may select dominated strategies as well and, in addition, not every Poisson game has a strictly perfect equilibrium.

On the other hand, as it happens in normal form games, not every undominated equilibrium is perfect. The same arguments that in normal form games suggest that we should dispose of some of the undominated equilibria that are not perfect are valid here. The difference being that, as argued above, some perfect equilibria may be dominated.

We define undominated perfect equilibria for Poisson games as strategy combinations that are limits of sequences of undominated equilibria of perturbed Poisson games. We prove that every Poisson game has at

\footnotetext{
${ }^{1}$ For instance, Myerson (2002), analyzing voting contexts, considers only Nash equilibria in which weakly dominated actions have been eliminated for all the types.
} 
least one undominated perfect equilibrium and that the set of undominated perfect equilibria is exactly the set of perfect equilibria which are also undominated.

Our analysis is focused on Poisson games. However, we must point out that none of the implications that we derive relies on the specific shape of the Poisson distribution. They are a consequence of a symmetry assumption that is embodied in the description of games of population uncertainty. Poisson games are taken as a reference because, together with their comparative computational simplicity, they are the most used subclass within this type of games.

This paper is organized as follows: In the next section we formally define Poisson games, strategies and Nash equilibria. We closely follow the description of Poisson games made by Myerson (1998). The third section is devoted to examine the properties of undominated strategies in Poisson games, where we show that there exist important asymmetries with respect to normal form games. The fourth section studies the perfect equilibrium concept and some of its possible variations. We define the concept of undominated perfect equilibrium for Poisson games in Section 5, where some of its properties are also proved.

\section{PRELIMINARIES}

Recall that a Poisson random variable is a discrete probability distribution characterized by only one parameter that coincides with the mean of the distribution. The probability that a Poisson random variable with mean $n$ takes the value $k$, being $k$ a nonnegative integer, is

$$
f(k ; n)=e^{-n} \frac{n^{k}}{k !} .
$$

A Poisson game $\Gamma$ is a five-tuple $(n, T, r, C, u)$. The number of players in the game is a Poisson random variable with parameter $n>0$. The set $T$ represents the set of possible types of players, we assume it to be a nonempty finite set.

As usual, if $A$ is a finite set, $\Delta(A)$ represents the set of probability distributions over $A$. Given the event that a player is in the game, she is of type $t \in T$ with probability $r(t)$. This information is contained in the vector $r \in \Delta(T)$. The decomposition property of the Poisson distribution implies that for each type $t$ in $T$, the number of players of the game whose type is $t$ is a Poisson random variable with parameter $n r(t)$. These random variables together are mutually independent and form a vector, called the type profile, which lists the number of players in the game who have each type. 
For any finite set $S$, we denote as $Z(S)$ the set of elements $w \in \mathbb{R}^{S}$ such that $w(s)$ is a nonnegative integer for all $s \in S$. Using this notation, the set $Z(T)$ denotes the set of possible values for the type profile in the game.

The set $C$ is the set of available choices or pure actions that a player may take. We assume that it is common to all players regardless of their type and that it is a finite set containing at least two different alternatives. The set $\Delta(C)$ is the set of mixed actions. Henceforth, we refer to mixed actions simply as actions.

The utility to each player depends on her type, on the action that she chooses and on the number of players, not counting herself, who choose each possible action. A vector that lists these numbers of players for each possible element of $C$ is called an action profile and belongs to the set $Z(C)$. We assume that preferences of a player of type $t$ can be summarized with a bounded function $u_{t}: C \times Z(C) \rightarrow \mathbb{R}$, i.e. $u_{t}(b, x)$ is the payoff that a player of type $t$ receives if she takes the pure action $b$ and the number of players who choose action $c$ is $x(c)$, for all $c \in C$. Furthermore, let $u=\left(u_{t}\right)_{t \in T}$.

In games with population uncertainty, as Myerson (1998, p. 377) argues, “... players' perceptions about each others' strategic behavior cannot be formulated as a strategy profile that assigns a randomized strategy to each specific individual of the game, because a player is not aware of the specific identities of all the other players". Notice that two players of the same type do not have any other known characteristic by which others can assess different conducts. The conclusion of the previous reasoning is that a strategy $\sigma$ is an element of $(\Delta(C))^{T}$, i.e. a mapping from the set of types to the set of possible actions. ${ }^{2}$

This symmetry assumption is a fundamental part of the description of the game. Notice that it is not made for convenience, on the contrary, symmetry is a critical assumption of a model of population uncertainty for it to be meaningful and well constructed.

If players play according to the strategy $\sigma, \sigma_{t}(c)$ is the probability that a player of type $t$ chooses the pure action $c$. The decomposition property of the Poisson distribution implies that the number of players of type $t \in T$ who choose the pure action $c$ is a Poisson distribution with parameter $n r(t) \sigma_{t}(c)$. The aggregation property of the Poisson distribution implies that any sum of independent Poisson random variables is also a Poisson random variable. It follows that the total number

\footnotetext{
${ }^{2}$ One may wonder how the game might be affected if the subdivision of types was finer, thus, allowing a larger variety of different behaviors. Myerson (1998) proves that, for Poisson games, utility-irrelevant subdivisions of types cannot substantially change the set of Nash equilibria (Theorem 4, page 386).
} 
of players who take the pure action $c$ is a Poisson distribution with parameter $n \tau(c)$, where $\tau(c)=\sum_{t \in T} r(t) \sigma_{t}(c)$.

A player of type $t$ who plays the pure action $b \in C$ while all other players are expected to play according to $\sigma$ has expected utility equal to

$$
U_{t}(b, \sigma)=\sum_{x \in Z(C)} \mathbb{P}(x \mid \sigma) u_{t}(b, x)
$$

where,

$$
\mathbb{P}(x \mid \sigma)=\prod_{c \in C} e^{-n \tau(c)} \frac{(n \tau(c))^{x(c)}}{x(c) !}
$$

and her expected utility from playing action $\theta \in \Delta(C)$ is

$$
U_{t}(\theta, \sigma)=\sum_{b \in C} \theta(b) U_{t}(b, \sigma)
$$

The set of best responses for a player of type $t$ against a strategy $\sigma$ is the set of actions that maximizes her expected utility given that the rest of the players, including those whose type is $t$, behave as prescribed by $\sigma$. The set $\operatorname{PBR}_{t}(\sigma)=\left\{c \in C: c \in \arg \max _{b \in C} U_{t}(b, \sigma)\right\}$ is the set of pure best responses against $\sigma$ for a player of type $t$. The set of mixed best responses against $\sigma$ for a player of type $t$ is the set of actions $\operatorname{BR}_{t}(\sigma)=\Delta\left(\operatorname{PBR}_{t}(\sigma)\right)$

Definition 1. The strategy $\sigma^{*}$ is a Nash equilibrium if $\sigma_{t}^{*} \in \mathrm{BR}_{t}\left(\sigma^{*}\right)$ for all $t$.

Standard fixed-point arguments show that every Poisson Game has at least one Nash equilibrium, see Myerson (1998).

\section{Dominated Strategies}

The admissibility principle, which in normal form games stipulates that no player must choose a dominated strategy, translates into the current framework imposing that no player should choose a dominated action. $^{3}$

\footnotetext{
${ }^{3}$ Some properties of (un)dominated strategies in normal form games are also true in Poisson games, although they have to be stated in terms of actions for Poisson games. It is easy to to see that if a pure action is dominated then every mixed action that gives positive probability to that pure action is also dominated, also that an action that does not give positive probability to a dominated pure action may be dominated, and that a pure action may just be dominated by a mixed action. See De Sinopoli and González Pimienta (2007).
} 
Definition 2. The action $\theta \in \Delta(C)$ is dominated for a player of type $t$ if there exists an alternative action $\theta^{\prime}$ such that $U_{t}(\theta, \sigma) \leq U_{t}\left(\theta^{\prime}, \sigma\right)$, for every possible strategy $\sigma$ and $U_{t}\left(\theta, \sigma^{\prime}\right)<U_{t}\left(\theta^{\prime}, \sigma^{\prime}\right)$ for at least one $\sigma^{\prime}$. A strategy $\sigma$ is dominated if there is some type $t$ for which $\sigma_{t}$ is a dominated action.

Although contained in a voting framework, Myerson (2002) offers a stronger definition of dominated action. Under such definition the (pure) action $c$ is dominated for a player of type $t$ if there exists an alternative (pure) action $b$ such that $u_{t}(c, x) \leq u_{t}(b, x)$ for every $x \in$ $Z(C)$ and with strict inequality for at least one $x^{\prime}$. This definition leads to too many undominated strategies. We use the former since it is equivalent to the definition of dominated strategies for normal form games.

We can use this formal apparatus to revisit the example discussed in the introduction. Let $a$ stand for "going out" and $b$ for "staying home":

Example 1. Let $\Gamma$ be a Poisson game with $n>0$, only one possible type, set of available choices $C=\{a, b\}$, and utility function:

$$
\begin{aligned}
& u(a, x)= \begin{cases}1 & \text { if } x(a)>0 \\
0 & \text { otherwise }\end{cases} \\
& u(b, x)=0 \quad \forall x \in Z(C) .
\end{aligned}
$$

Since this Poisson game has only one possible type, we can identify the set of strategies with the set of actions. There are two equilibria, $a$ and $b$. We have already argued that the equilibrium strategy $b$ is unsatisfactory. Notice that $b$ is a dominated action, even when we consider the stronger definition given by Myerson (2002), which makes $b$ a dominated strategy.

The example highlights that the Nash equilibrium concept is inadequate for Poisson games since it allows for equilibrium points where players use dominated actions (strategies).

In normal form games the admissibility requirement is taken care of by perfection. Every perfect equilibrium selects only undominated strategies and, moreover, perfect equilibrium conditions do not admit just every equilibrium in undominated strategies, but only a subset of them.

Mertens (2004) links undominated strategies and perfect equilibrium through the concept of admissibility. He defines 3 possible concepts of admissible best response: 
$(\alpha) \theta$ is an admissible best response against $\sigma$ if there exists a sequence of completely mixed $\sigma^{k}$ converging to $\sigma$ such that $\theta$ is a best response against each $\left(\sigma^{k}\right)$.

( $\beta$ ) $\theta$ is an admissible best response against $\sigma$ if $\theta$ is a best response against $\sigma$ and there exist completely mixed $\sigma^{\prime}$ such that $\theta$ is a best response against $\sigma^{\prime}$.

$(\gamma) \theta$ is an admissible best response against $\sigma$ if $\theta$ is a best response against $\sigma$ and no other best response $\theta^{\prime}$ is at least as good against every $\sigma^{\prime}$ and better against some.

The third concept corresponds to the usual concept of admissibility, i.e. players must use undominated strategies, while the first one is a characterization of perfect equilibria. In normal form games, the first concept is strictly stronger than the second, which in turn is strictly stronger than the third.

It is not clear that the same relationship holds for Poisson games (apart from the fact that the second concept is clearly weaker than the first). We are interested in finding out if there is any connection between $\alpha$ and $\gamma$ in the present setting. Once we know this, we will be able to propose a definition of a strong version of admissibility for Poisson games.

This is done in Section 5. Before that we have to extend the perfect equilibrium concept to Poisson games and look into its properties.

\section{Perfection}

Three equivalent definitions of perfect equilibrium have been proposed for normal form games. One based on perturbed games (Selten, 1975), a second one based on the item $\alpha$ of the previous list (also Selten, 1975) and a last one based on $\varepsilon$-perfect equilibria (Myerson, 1978). Below we provide the three corresponding definitions for Poisson games and prove their equivalence, so that we always have the most advantageous definition available.

The leading definition that we use is the one based on perturbed games

Definition 3. Let $\Gamma$ be a Poisson Game, for every $t \in T$, let $\eta_{t}$ and $\Sigma_{t}\left(\eta_{t}\right)$ be defined by:

$$
\begin{aligned}
& \eta_{t} \in \mathbb{R}^{C} \text { with } \eta_{t}(c)>0 \text { for all } c \in C \text { and } \sum_{c \in C} \eta_{t}(c)<1 \\
& \Sigma_{t}\left(\eta_{t}\right)=\left\{\theta \in \Delta(C): \theta(c) \geq \eta_{t}(c) \text { for all } c \in C\right\} .
\end{aligned}
$$


Furthermore, let $\eta=\left(\eta_{t}\right)_{t}$. The perturbed Poisson game $(\Gamma, \eta)$ is the Poisson game $(n, T, r, C, u)$ where players of type $t$ are restricted to play only actions in $\Sigma_{t}\left(\eta_{t}\right)$, for every $t$.

In the perturbed Poisson game $(\Gamma, \eta)$, an action $\theta \in \Sigma_{t}\left(\eta_{t}\right)$ is a best reply against $\sigma \in \Sigma(\eta)=\prod_{t \in T} \Sigma_{t}\left(\eta_{t}\right)$ for a player of type $t$ if every pure action $c$ that is not a best response in $\Gamma$ against $\sigma$ for a player of type $t$ is played with minimum probability, that is to say, $\sigma_{t}(c)=\eta_{t}(c)$. A strategy $\sigma \in \Sigma(\eta)$ is an equilibrium of the Poisson game $(\Gamma, \eta)$ if for every type $t, \sigma_{t}$ is a best response to $\sigma$ in $(\Gamma, \eta)$. Kakutani fixed point theorem implies that:

Lemma 1. Every perturbed Poisson game has an equilibrium.

Perturbed games lead to the following definition of perfection:

Definition 4. A strategy $\sigma$ is a perfect equilibrium if it is the limit point of a sequence $\left\{\sigma^{\eta}\right\}_{\eta \rightarrow 0}$, where $\sigma^{\eta}$ is an equilibrium of the perturbed game $(\Gamma, \eta)$, for all $\eta$.

Since every perturbed Poisson game has an equilibrium and since this equilibrium is contained in the compact set $(\Delta(C))^{T}$, every Poisson game has a perfect equilibrium. ${ }^{4}$ By continuity of the utility function, every perfect equilibrium is also a Nash equilibrium.

As we mentioned earlier, another possible definition of perfect equilibrium uses $\varepsilon$-perfect equilibria. A completely mixed strategy $\sigma^{\varepsilon}$ is an $\varepsilon$-perfect equilibrium if it satisfies:

$$
U_{t}\left(c, \sigma^{\varepsilon}\right)<U_{t}\left(d, \sigma^{\varepsilon}\right) \text {, then } \sigma_{t}^{\varepsilon}(c) \leq \varepsilon \text { for all } t \in T .
$$

What follows is an adaption to Poisson games of some results and proofs contained in van Damme (1991, pp. 26-29) for perfect equilibrium in normal form games. Although this is rather straightforward, we include it here to maintain the paper self-contained. The next lemma lists the two remaining concepts of perfect equilibrium and proves their equivalence.

Lemma 2. Let $\Gamma$ be a Poisson game, and let $\sigma \in(\Delta(C))^{T}$. The following assertions are equivalent:

(1) $\sigma$ is a perfect equilibrium of $\Gamma$,

(2) $\sigma$ is a limit point of a sequence $\left\{\sigma^{\varepsilon}\right\}_{\varepsilon \rightarrow 0}$, where $\sigma^{\varepsilon}$ is an $\varepsilon$-perfect equilibrium of $\Gamma$, for all $\varepsilon$, and

\footnotetext{
${ }^{4}$ Take any sequence of $\eta \rightarrow 0$, and for each $\eta$, an equilibrium $\sigma^{\eta}$ of $(\Gamma, \eta)$. The sequence $\left\{\sigma^{\eta}\right\}_{\eta \rightarrow 0}$ has a convergent subsequence whose limit point is a perfect equilibrium.
} 
(3) $\sigma$ is a limit point of a sequence $\left\{\sigma^{\varepsilon}\right\}_{\varepsilon \rightarrow 0}$ of completely mixed strategy combinations with the property that, for all $t, \sigma_{t}$ is a best response against each element $\sigma^{\varepsilon}$ in this sequence.

Proof. (1) $\rightarrow(2)$ : Let $\sigma$ be a limit point of a sequence $\left\{\sigma^{\eta}\right\}_{\eta \rightarrow 0}$, where $\sigma^{\eta}$ is equilibrium of $\Gamma(\eta)$ for all $\eta$. Define $\varepsilon(\eta) \in \mathbb{R}_{++}$by

$$
\varepsilon(\eta)=\max _{t, c} \eta_{t}(c) \text {. }
$$

Then $\sigma^{\eta}$ is an $\varepsilon(\eta)$-perfect equilibrium for $\Gamma$.

$(2) \rightarrow(3)$ : Let $\left\{\sigma^{\varepsilon}\right\}_{\varepsilon \rightarrow 0}$ be a sequence of $\varepsilon$-perfect equilibria with limit $\sigma$. By continuity, every element of the carrier of $\sigma$, which from now on we denote as $\mathcal{C}(\sigma)$, is a best response against $\sigma(\varepsilon)$ for $\varepsilon$ close enough to zero.

$(3) \rightarrow(1)$ : Let $\left\{\sigma^{\varepsilon}\right\}_{\varepsilon \rightarrow 0}$ be a sequence as in (3) with limit $\sigma$. Define $\eta^{\varepsilon}$ by:

$$
\eta_{t}^{\varepsilon}(c)=\left\{\begin{array}{ll}
\sigma_{t}^{\varepsilon}(c) & \text { if } c \notin C\left(\sigma_{t}\right) \\
\varepsilon & \text { otherwise }
\end{array} \text { for all } t, c .\right.
$$

For $\varepsilon$ small enough $\sigma^{\varepsilon}$ is equilibrium of the perturbed Poisson game $\left(\Gamma, \eta^{\varepsilon}\right)$, which establishes (1).

Example 2. Let $\Gamma$ be a Poisson game with expected number of players equal to $n=2,{ }^{5}$ only one possible type, set of choices $C=\{a, b\}$, and utility function

$$
\begin{aligned}
& u(a, x)=e^{-2} \quad \forall x \\
& u(b, x)= \begin{cases}1 & \text { if } x(a)=x(b)=1 \\
0 & \text { otherwise. }\end{cases}
\end{aligned}
$$

Notice that $e^{-2}$ is the probability that $x(a)=x(b)=1$ under the strategy $\sigma=1 / 2 a+1 / 2 b$. Also notice that the action $b$ is dominated by the action $a$, the former only does as good as the latter against the strategy $\sigma=1 / 2 a+1 / 2 b$, and does strictly worse for any other strategy $\sigma^{\prime} \neq \sigma$. The action $\theta=1 / 2 a+1 / 2 b$ is also dominated by $a$. Nevertheless it is a best response against the strategy $\sigma$. Consequently, the dominated strategy $\sigma$ is a perfect equilibrium.

\footnotetext{
${ }^{5}$ The set of examples in the paper is designed to be as clear and simple as possible. This is the reason why we many times fix the expected number of players to be $n=1$ or $n=2$. This contrasts with the fact that Poisson games fit more naturally to a situation where the expected number of players is large. At the expense of computational simplicity, similar examples can be constructed for any value of the Poisson parameter $n$.
} 
The next example is more illustrative in showing how the perfect equilibrium concept fails to select only undominated strategies in Poisson games.

Example 3. Consider the Poisson game $\Gamma=\{n, T, r, C, u\}$, with expected number of players $n=2$, set of types $T=\{1,2\}$, with equal probability for each type $r(1)=r(2)=1 / 2$, set of choices $C=\{a, b\}$, and utility function:

$$
\begin{aligned}
& u_{1}(a, x)= \begin{cases}1 & \text { if } x(b)=1 \\
0 & \text { otherwise }\end{cases} \\
& u_{1}(b, x)=e^{-1} \quad \forall x \in Z(C) \\
& u_{2}(a, x)=e^{-1} \quad \forall x \in Z(C) \\
& u_{2}(b, x)= \begin{cases}1 & \text { if } x(a)=1 \\
0 & \text { otherwise. }\end{cases}
\end{aligned}
$$

The number of players of type 1 is a Poisson random variable with expected value equal to 1 . The same is true for type 2. Notice also that $e^{-1}$ coincides with the probability that a Poisson random variable of parameter 1 is equal to 1 . The action $a$ is dominated for players of type 1 , while action $b$ is dominated for players of type 2 . We claim that the strategy $\sigma=\left(\sigma_{1}, \sigma_{2}\right)=(a, b)$ is a perfect equilibrium. Take the sequence of $\varepsilon$-perfect equilibria $\sigma_{1}^{\varepsilon}=(1-\varepsilon) a+\varepsilon b, \sigma_{2}^{\varepsilon}=\varepsilon a+(1-\varepsilon) b$. For every $\varepsilon, U_{t}\left(a, \sigma^{\varepsilon}\right)=U_{t}\left(b, \sigma^{\varepsilon}\right)$, and the sequence $\left\{\sigma^{\varepsilon}\right\}_{\varepsilon \rightarrow 0}$ converges to $\sigma$.

Each one of this last two examples actually proves the next proposition: ${ }^{6}$

Proposition 1. A Perfect equilibrium can be dominated.

\footnotetext{
${ }^{6}$ As we mentioned in the introduction, the results are a consequence of the symmetry assumption that is inherent to games of population uncertainty. To see why, consider the following three person normal form game:

$$
\begin{array}{ccccccc} 
& a & b & & a & b \\
a & 1,1,1 & 0,0,0 & & a & 0,0,0 & 1,1,1 \\
b & 0,0,0 & 1,1,1 & & b & 1,1,1 & 0,0,0 \\
& \multicolumn{2}{c}{a} & & \multicolumn{2}{c}{b}
\end{array}
$$

There are two symmetric equilibria, one in which every player plays $a$ and a second one in which every player plays $1 / 2 a+1 / 2 b$. Both are perfect equilibria and, hence, undominated. However, if dominated strategies are defined over symmetric strategies of the opponents, strategy $b$ and, consequently, strategy $1 / 2 a+1 / 2 b$ are dominated for every player.
} 
Hence, the doubts that we have raised at the end of the previous section are justified. In Poisson games, the relationship between $\alpha$ and $\gamma$ of the possible concepts of admissible best response listed by Mertens is different from the one that holds in normal form games.

In the last example, the undominated equilibrium $\sigma=\left(\sigma_{1}, \sigma_{2}\right)=$ $(b, a)$ is also perfect. The next question that we must answer is whether or not undominated equilibria are always perfect. Proposition 2 shows that in this case things work as they do in normal form games.

Proposition 2. An undominated equilibrium may not be perfect.

Proof. Consider a Poisson game $\Gamma$, with expected number of players equal to $n$, two possible types with equal probabilities, i.e. $T=\{1,2\}$ and $r(1)=r(2)=1 / 2$, set of available choices $C=\{a, b, c\}$ and utility function: ${ }^{7}$

$$
\begin{array}{ll}
u_{1}(a, x)=x(a)+x(b) & \\
u_{1}(b, x)=|x(a)+x(b)-x(c)| & \\
u_{1}(c, x)=0 & \forall x \in Z(C) \\
& \\
u_{2}(a, x)=x(a) & \forall x \in Z(C) \\
u_{2}(b, x)=0 & \forall x \in Z(C) . \\
u_{2}(c, x)=0 &
\end{array}
$$

The game has a continuum of undominated equilibria $(\lambda a+(1-\lambda) b, a)$, for $\lambda$ taking values in the closed interval $[0,1]$. Note, in particular, that the action $b$ is not dominated for players of type 1 since it does better than the action $a$ against the strategy $\sigma=\left(\sigma_{1}, \sigma_{2}\right)=(c, c)$. However, the strategy $\hat{\sigma}=\left(\hat{\sigma}_{1}, \hat{\sigma}_{2}\right)=(a, a)$ is the unique perfect equilibrium of the game.

The example used in the proof of the last proposition depicts that there may be unreasonable equilibria in undominated strategies. Consider the strategy $\sigma^{\prime}=(\lambda a+(1-\lambda) b, a)$ with $\lambda \in[0,1)$. It is difficult to justify that a player of type 1 will stick to the prescribed strategy. A rational player should not risk his equilibrium payoff, even more when there is no possible expected benefit from such behavior. Suppose there

\footnotetext{
${ }^{7}$ Notice that the utility functions that we use in this example, and in some of the following ones are not bounded, as we assumed in the general description of Poisson games made in Section 2. The main features of all the examples discussed are preserved if we put an upper bound on utilities, that is to say, if utilities are given by $\tilde{u}_{t}(y, x)=\min \left\{u_{t}(y, x), K\right\}$, where $K$ is a sufficiently large number with respect to $n$. However, we maintain the unbounded functions for the sake of simplicity.
} 
was an unexpected deviation from $\sigma^{\prime}$ toward $c$, placing weight in the action $b$ would pay off to players of type 1 if and only if such a deviation was drastic and it would hurt otherwise.

Since not all perfect equilibria are undominated and not all undominated equilibria are perfect, we would like to have available an equilibrium concept that implies both. At this early stage, we do not want to go very far apart from the perfect equilibrium concept. We notice, nevertheless, that the equilibrium discussed in Example 3 is also proper, for a straightforward extension of this concept to Poisson games ${ }^{8}$ since every player has only two possible choices. ${ }^{9}$ Strictly perfect equilibrium, does not help either. As argued above, the strategy $\sigma=1 / 2 a+1 / 2 b$ is an equilibrium of the Poisson game described in

${ }^{8} \mathrm{~A}$ completely mixed strategy $\sigma^{\varepsilon}$ is an $\varepsilon$-proper equilibrium if it satisfies:

$$
U_{t}\left(c, \sigma^{\varepsilon}\right)<U_{t}\left(d, \sigma^{\varepsilon}\right) \text {, then } \sigma_{t}^{\varepsilon}(c) \leq \varepsilon \sigma_{t}^{\varepsilon}(d) \text { for all } t \in T \text {. }
$$

A strategy $\sigma$ is proper if it is a limit point of a sequence $\left\{\sigma^{\varepsilon}\right\}_{\varepsilon \rightarrow 0}$, where $\sigma^{\varepsilon}$ is an $\varepsilon$-proper equilibrium of $\Gamma$, for all $\varepsilon$.

${ }^{9}$ As it should be expected, not every perfect equilibrium is proper. Consider the Poisson game $\Gamma=\{n, T, r, C, u\}$, with expected number of players $n=2$, two possible types that are equally probable, i.e. $T=\{1,2\}$ and $r(1)=r(2)=1 / 2$, set of choices $C=\{a, b, c, d\}$ and utility function:

$$
\begin{array}{ll}
u_{1}(x, a)=0 & \forall x \\
u_{1}(x, b)=x(d)-x(c) \\
u_{1}(x, c)=-1 & \forall x \\
u_{1}(x, d)=-2 & \forall x \\
u_{2}(x, a)= \begin{cases}1 & \text { if } x(b)=1 \\
0 & \text { otherwise }\end{cases} \\
u_{2}(x, b)=e^{-1} & \forall x \\
u_{2}(x, c)=-1 & \forall x \\
u_{2}(x, d)=-2 & \forall x
\end{array}
$$

The action $a$ is dominated for players of type 2 by action $b$. The strategy $\sigma=$ $\left(\sigma_{1}, \sigma_{2}\right)=(b, a)$ is perfect. To see this consider the sequence of $\varepsilon$-perfect equilibria:

$$
\begin{aligned}
& \sigma_{1}^{\varepsilon}=\frac{1}{3} \varepsilon a+(1-\varepsilon) b+\frac{1}{3} \varepsilon c+\frac{1}{3} \varepsilon d \\
& \sigma_{2}^{\varepsilon}=\left(1-\varepsilon-2 \varepsilon^{2}\right) a+\varepsilon b+\varepsilon^{2} c+\varepsilon^{2} d
\end{aligned}
$$

For every type, action $d$ is always strictly worse than actions $c$, hence, in any $\varepsilon$-proper equilibrium, the former is played with strictly less probability than the latter. Therefore, a player of type 1 plays the action $b$ with a probability less than $\varepsilon$ times the probability that she gives to $a$. Hence, in no proper equilibrium she plays $b$ with positive probability. 
Example 2. Notice that this equilibrium uses completely mixed strategies, and consequently, it is a strictly perfect equilibrium (again, using a straightforward extension of the concept to Poisson games). ${ }^{10}$

Examples 2 and 3 suggest that we may also demand robustness against perturbations other than trembles. (In Example 2, the payoff $e^{-2}$ coincides with the probability that $x(a)=x(b)=1$ under the strategy $\sigma=1 / 2 a+1 / 2 b$. In example 3 the payoff $e^{-1}$ coincides with the probability that $x(a)=1$, also that $x(b)=1$, under the strategy $\sigma=\left(\sigma_{1}, \sigma_{2}\right)=(a, b)$.) Specifically, perturbations in the Poisson parameter $n$ seem like the natural candidate as the model is of population uncertainty. Let us study the following equilibrium concept.

Definition 5. The strategy $\sigma$ is a perfect* equilibrium of the Poisson game $\Gamma=(n, T, r, C, u)$ if there exists a $\xi>0$ such that $\sigma$ is a perfect equilibrium of the Poisson game $\tilde{\Gamma}=(\tilde{n}, T, r, C, u)$ for all $\tilde{n} \in(n-$ $\xi, n+\xi)$.

A perfect* equilibrium is a perfect equilibrium, not only of the original game, but also of every game that is obtained by small perturbations in the expected number of players. Notice that we cannot rely exclusively on perturbations in the expected numbers of players. One can easily construct examples that do not pose any restriction in the number of players with unreasonable Nash equilibria. See for instance Example 1.

Let us analyze why the perfect* equilibrium concept is not adequate by means of the following example.

Example 4. Consider the family of Poisson games with expected number of players equal to $n>\frac{4}{7},{ }^{11}$ with only one type, set of choices

\footnotetext{
${ }^{10}$ In addition strictly perfect equilibrium does not satisfy existence. To see this, consider a Poisson game with expected number of players $n>0$, only one possible type, four different choices $C=\{a, b, c, d\}$ and utility function:

$$
\begin{array}{ll}
u(a, x)=1+x(c) \\
u(b, x)=1+x(d) \\
u(c, x)=0 & \forall x \\
u(d, x)=0 & \forall x .
\end{array}
$$

Notice that there is no equilibrium that is "robust" to every possible tremble.

${ }^{11}$ It is enough that $n$ is such that $\ln n>-n$.
} 
$C=\{a, b\}$, and utility function:

$$
\begin{aligned}
& u(a, x)=x(b) \\
& u(b, x)= \begin{cases}1 & \text { if } x(a)=x(b)=0 \\
2 x(a) & \text { otherwise. }\end{cases}
\end{aligned}
$$

Every game has a unique equilibrium and it depends on $n .{ }^{12}$ Consequently, it does not have a perfect* equilibrium.

This example prompts us to discard the previous equilibrium concept and reveals that demanding stability against variations in the Poisson parameter $n$ forces to tolerate, at least, smooth variations of the equilibrium strategy if we want to retain existence. Therefore, if $\sigma$ is a perfect equilibrium of $\Gamma$, we may want any game that only differs from $\Gamma$ in that it has a slightly different number of expected players to have a perfect equilibrium that is not far away from $\sigma$.

As the next example shows, this relaxation would bring back dominated equilibria.

Example 5. Let $\Gamma$ be a Poisson game with expected number of players equal to $n=6$, two different types $T=\{1,2\}$ with $r(1)=2 / 3$ and $r(2)=1 / 3$, set of available choices $C=\{a, b, c, d\}$, and utility function:

$$
\begin{aligned}
& u_{1}(h, x)=0 \quad \forall x \in Z(C), \forall h \in C \\
& u_{2}(a, x)= \begin{cases}1 & \text { if } x(c)=x(d)=1 \\
0 & \text { otherwise }\end{cases} \\
& u_{2}(b, x)=e^{-2} \quad \forall x \in Z(C) \\
& u_{2}(h, x)=-1 \quad \forall x \in Z(C), h=c, d .
\end{aligned}
$$

Notice first that the number of players with type 1 is a Poisson random variable of parameter 4 . The strategy $\sigma=\left(\sigma_{1}, \sigma_{2}\right)=(1 / 4 a+1 / 4 b+$ $1 / 4 c+1 / 4 d, a)$ implies that the event $x(c)=x(d)=1$ occurs with probability $e^{-2}$. The strategy $\sigma$ is a perfect equilibrium where players of type 2 play dominated strategies. Take $g$ to be a small number. The Poisson game $\Gamma^{g}=\{n+g, T, r, C, u\}$ has a dominated perfect equilibrium very close to $\sigma$ where players of type 1 play action $(1 / 4+$ $\kappa, 1 / 4+\kappa, 1 / 4-\kappa, 1 / 4-\kappa)$, for $\kappa=g /(24+4 g)$, and players of type 2 play action $a$. On the other hand, the Poisson game $\Gamma^{g}=\{n-$ $g, T, r, C, u\}$ also has a dominated perfect equilibrium very close to $\sigma$, where players of type 1 play action $\left(1 / 4-\kappa^{\prime}, 1 / 4-\kappa^{\prime}, 1 / 4+\kappa^{\prime}, 1 / 4+\kappa^{\prime}\right)$, for $\kappa^{\prime}=g /(24-4 g)$, and players of type 2 play action $a$.

\footnotetext{
${ }^{12}$ The unique equilibrium is $\sigma=\alpha a+(1-\alpha) b$, where $\alpha=\left(1-\frac{1}{n e^{n}}\right) /\left(3-\frac{2}{e^{n}}\right)$.
} 
So far we have provided a number of results and examples that show that some equilibrium concepts proposed for normal form games do not retain either admissibility or existence when extended to Poisson games. In the next section we propose an equilibrium concept that shows that, in this setting, these properties are not incompatible.

\section{Undominated Perfect Equilibria}

The same arguments that in normal form games compel to dispose of the undominated equilibria that are not perfect are also well founded here. Perfection is a weak requirement, it asks for stability against one single perturbation, not against every possible perturbation. As a result, equilibria that are not perfect are very unstable.

The main difference in the current setting is that there are perfect equilibria that are not undominated. We want to put forward a strong version of admissibility for games with population uncertainty. Such a definition comprises items $\alpha$ and $\gamma$ from the list of possible concepts of admissibility provided by Mertens (2004) and listed at the end of Section 3.

Definition 6. $\theta$ is an admissible best response against $\sigma$ if it is undominated and there exists a sequence of completely mixed $\sigma^{k}$ converging to $\sigma$ such that $\theta$ is a best response against each $\left(\sigma^{k}\right)$.

Accordingly, we may say that the strategy $\sigma$ is admissible if for every $t, \sigma_{t}$ is an admissible best response against $\sigma$. Therefore, if $\sigma$ is an admissible strategy it is a perfect equilibrium, and we may talk about the set of admissible equilibria.

We want to propose an equilibrium concept that satisfies admissibility and that generates a nonempty set of equilibria for any game. Such a concept is introduced in Definition 7, the admissibility property will come directly from the definition and the existence result is offered in Proposition 4. The following Proposition shows that every Poisson game has an equilibrium in undominated strategies. It could have been proposed as a corollary of our main existence result. However, we prefer to invert the order of presentation so that the argument of the main proof can be more easily followed.

We proceed to prove that every Poisson game has an equilibrium in undominated strategies. Notice that the set of undominated strategies is not convex and, hence, we could not show existence of undominated equilibria using a standard fixed point argument in this set. A constructive proof shows that: 
Proposition 3. Every Poisson game has a Nash equilibrium in undominated strategies.

Proof. Consider a Poisson game $\Gamma$, with set of choices $C$ and utility vector $u$. Recall that if $\theta$ is an action, $\mathcal{C}(\theta)$ denotes the carrier of $\theta$. Notice that if $\mathcal{C}(\theta) \subseteq \mathcal{C}\left(\theta^{\prime}\right)$ then there exist a $\lambda \in(0,1)$ and an action $\theta^{\prime \prime}$ such that $\theta^{\prime}=\lambda \theta+(1-\lambda) \theta^{\prime \prime}$. If $\theta$ is dominated for players of type $t$, there exists a $\tilde{\theta}$ that dominates it, and a $\hat{\sigma}$ such that $U_{t}(\theta, \hat{\sigma})<U_{t}(\tilde{\theta}, \hat{\sigma})$. Moreover, if $\mathcal{C}(\theta) \subseteq \mathcal{C}\left(\theta^{\prime}\right)$ then $\theta^{\prime}=\lambda \theta+(1-\lambda) \theta^{\prime \prime}$ is dominated by $\tilde{\theta}^{\prime}=\lambda \tilde{\theta}+(1-\lambda) \theta^{\prime \prime}$ and $U_{t}\left(\theta^{\prime}, \hat{\sigma}\right)<U_{t}\left(\tilde{\theta}^{\prime}, \hat{\sigma}\right)$.

This implies that we can talk about dominated carriers and that, given a dominated carrier $\mathcal{C}$ there exists a strategy $\hat{\sigma}$ such that any action with carrier that contains $\mathcal{C}$ is dominated by an action that is a strictly better response to $\hat{\sigma}$.

Consider the set of all possible carriers, and call $D_{t}$ the finite set of all dominated carriers for players of type $t$. For each minimal element of $D_{t}$, say $d_{t}$, let $\sigma_{d_{t}}$ be a strategy such that any action with carrier that contains $d_{t}$ is dominated by an action that is a strictly better response to such a strategy. Let $M_{t}$ be the set of minimal elements of $D_{t}$.

For $\lambda>0$, define a new Poisson game $\Gamma^{\lambda}$, with utility vector given by

$$
u_{t}^{\lambda}(c, x)=u_{t}(c, x)+\lambda \sum_{d_{t} \in M_{t}} U_{t}\left(c, \sigma_{d_{t}}\right)
$$

which implies expected utilities,

$$
U_{t}^{\lambda}\left(\theta_{t}, \sigma\right)=U_{t}\left(\theta_{t}, \sigma\right)+\lambda \sum_{d_{t} \in M_{t}} U_{t}\left(\theta_{t}, \sigma_{d_{t}}\right) .
$$

This new Poisson game has an equilibrium. Moreover, no dominated action of the original game is used with positive probability in that equilibrium. Take a sequence of $\lambda \rightarrow 0$. There exists a subsequence of equilibria $\left\{\sigma^{\lambda}\right\}_{\lambda}$ that converges to some $\bar{\sigma}$. By continuity of the utility function, $\bar{\sigma}$ is an equilibrium in undominated strategies of the original game.

In section 4 we have defined perturbed Poisson games. In a perturbed game $(\Gamma, \eta)$ an action $\theta \in \Sigma_{t}\left(\eta_{t}\right)$ is dominated for type $t$ if there exists an alternative action $\theta^{\prime} \in \Sigma_{t}\left(\eta_{t}\right)$ such that $U_{t}(\theta, \sigma) \leq U_{t}\left(\theta^{\prime}, \sigma\right)$, for every possible strategy $\sigma \in \Sigma(\eta)$ and $U_{t}\left(\theta, \sigma^{\prime}\right)<U_{t}\left(\theta^{\prime}, \sigma^{\prime}\right)$ for at least one $\sigma^{\prime} \in \Sigma(\eta)$.

We could strength the definition of perfection (Definition 4), asking the equilibria in the sequence to be undominated: 
Definition 7. A strategy $\sigma$ is an undominated perfect equilibrium of a Poisson game $\Gamma$ if it is the limit point of a sequence $\left\{\sigma^{\eta}\right\}_{\eta \rightarrow 0}$ where $\sigma^{\eta}$ is an undominated equilibrium of $(\Gamma, \eta)$ for all $\eta$.

Every perturbed Poisson game has an undominated equilibrium. ${ }^{13}$ Moreover, for $\eta$ close to zero the sets of dominated carriers in $\Gamma$ and in $(\Gamma, \eta)$ coincide for every possible type. Hence, every undominated perfect equilibrium is perfect and undominated (i.e., it satisfies our strong version of admissibility). Since every perturbed Poisson game has an undominated equilibrium and since this equilibrium is contained in the compact set $(\Delta(C))^{T}$ it follows: ${ }^{14}$

Proposition 4. Every Poisson game has an undominated perfect equilibrium.

The definition appears to be stronger than requiring separately perfect equilibrium and undominated strategies because it poses restrictions in the sequence of equilibria of the associated perturbed Poisson games. The next Proposition shows that both definitions are equivalent. This fact, in view of Lemma 2, simplifies the analysis of undominated perfect equilibrium in Poisson games.

Proposition 5. The set of undominated perfect equilibria coincides with the intersection of the set of undominated equilibria with the set of perfect equilibria.

Proof. Let $\sigma$ belong both to the set of perfect equilibria and to the set of undominated equilibria of $\Gamma$. Since $\sigma$ is perfect it is the limit point of a sequence $\left\{\sigma^{\eta}\right\}_{\eta \rightarrow 0}$ where $\sigma^{\eta}$ is an equilibrium of $(\Gamma, \eta)$. Because $\sigma$ is undominated, its carrier is not a dominated one. Moreover, for $\eta$ close to zero the sets of dominated carriers in $\Gamma$ and in $(\Gamma, \eta)$ coincide for every possible type. For each $\eta$, let $\eta^{\prime}$ be defined by:

$$
\eta_{t}^{\prime}(c)=\left\{\begin{array}{ll}
\sigma_{t}^{\eta}(c) & \text { if } \sigma_{t}(c)=0 \\
\eta_{t}(c) & \text { otherwise }
\end{array} \text { for all } c, t .\right.
$$

Then $\sigma^{\eta^{\prime}}=\sigma^{\eta}$ is an undominated equilibrium of $\left(\Gamma, \eta^{\prime}\right)$. Moreover the sequence of $\eta^{\prime}$ converges to zero. Hence, $\sigma$ is the limit point of the sequences $\left\{\sigma^{\eta^{\prime}}\right\}_{\eta^{\prime} \rightarrow 0}$ of undominated equilibria for $\left(\Gamma, \eta^{\prime}\right)$.

\footnotetext{
${ }^{13}$ To see this, a modification of the proof of Proposition 3 would do, where the carrier of an action is defined as the set of pure actions that receive strictly more probability than the minimum weight imposed by $\eta$.

${ }^{14}$ See footnote 4 .
} 


\section{REFERENCES}

De Sinopoli, F., González Pimienta, C., Apr. 2007. Undominated (and) perfect equilibrium in poisson games. Economics Working Paper Series we073117, Universidad Carlos III, Departamento de Economía.

Mertens, J., 2004. Ordinality in non cooperative games. Int. J. Game Theory 32 (3), 387-430.

Milchtaich, I., May 2004. Random-player games. Games Econ. Behav. 47 (2), 353-388.

Myerson, R., 1978. Refinements of the Nash equilibrium concept. Int. J. Game Theory 7 (2), 73-80.

Myerson, R., 1998. Population uncertainty and Poisson games. Int. J. Game Theory 27 (3), 375-392.

Myerson, R., 2000. Large Poisson Games. J. Econ. Theory 94 (1), 7-45.

Myerson, R., 2002. Comparison of Scoring Rules in Poisson Voting Games. J. Econ. Theory 103 (1), 219-251.

Selten, R., 1975. Re-examination of the perfectness concept for equilibrium points in extensive games. Int. J. Game Theory 4, 24-55.

van Damme, E., 1991. Stability and Perfection of Nash Equilibria. Springer-Verlag, Berlin. 